\title{
Optimal reinsurance with general risk measures
}

\author{
Alejandro Balbás ${ }^{\mathrm{a}}$, Beatriz Balbás ${ }^{\mathrm{a}}$, Antonio Heras ${ }^{\mathrm{b}, *}$ \\ ${ }^{a}$ University Carlos III of Madrid, Department of Business Economics, CL, Madrid 126, 28903 Getafe, Madrid, Spain \\ ${ }^{\mathrm{b}}$ University Complutense of Madrid, Somosaguas-Campus, 28223 Pozuelo de Alarcón, Madrid, Spain
}

\section{A R T I C L E I N F O}

\section{Article history:}

Received February 2008

Received in revised form

November 2008

Accepted 18 November 2008

\section{JEL classification:}

G22

G11

MSC:

$91 \mathrm{~B} 30$

91B28

$90 C 48$

Keywords:

Optimal reinsurance

Risk measure and deviation measure

Optimality conditions

\begin{abstract}
A B S T R A C T
This paper studies the optimal reinsurance problem when risk is measured by a general risk measure. Necessary and sufficient optimality conditions are given for a wide family of risk measures, including deviation measures, expectation bounded risk measures and coherent measures of risk. The optimality conditions are used to verify whether the classical reinsurance contracts (quota-share, stop-loss) are optimal essentially, regardless of the risk measure used. The paper ends by particularizing the findings, so as to study in detail two deviation measures and the conditional value at risk.
\end{abstract}

\section{Introduction}

General risk measures are becoming more and more important in insurance and finance. The study of risk measures beyond the variance has a long history in Actuarial Sciences, and probably in Statistics. Early accounts in Actuarial Science include Bühlmann (1970), Gerber (1979) and Goovaerts et al. (1984). Early work in Probability and Statistics includes Huber (1981) and Schmeidler (1986).

The paper by Artzner et al. (1999) on coherent measures of risk gave a new impulse to this topic and, since then, many authors have further extended the discussion. The recent development of new markets (insurance or weather linked derivatives, commodity or energy/electricity derivatives etc.) and products (inflationlinked bonds, equity indexes annuities, hedge funds etc.), the necessity of managing new types of risk (credit risk, operational risk etc.), the presence of asymmetries and fat tails, and the (often legal) obligation of providing initial capital requirements, have significantly increased the importance of finding proper

\footnotetext{
* Corresponding author.

E-mail addresses: alejandro.balbas@uc3m.es (A. Balbás),

beatriz.balbas@uc3m.es (B. Balbás), aheras@ccee.ucm.es (A. Heras)
}

measures of risk and risk management techniques. ${ }^{1}$ Hence, it is not surprising that the recent literature presents many interesting contributions focusing on new methods for measuring risk. Among others, Goovaerts et al. (2004a) have introduced the consistent risk measures, also studied in Burgert and Rüschendorf (2006), Goovaerts et al. (2004b) and Goovaerts and Laeven (2008) have introduced mixtures of exponentials, Frittelli and Scandolo (2005) have analyzed risk measures for stochastic processes, and Rockafellar et al. (2006) have defined the deviations and the expectation bounded risk measures.

Many classical actuarial and financial problems have been revisited using risk measures beyond the variance. For example, Laeven and Goovaerts (2004) and Dhaene et al. (2008) analyze the capital allocation problem, Carr et al. (2001) and Nakano (2004) draw on risk measures to price in incomplete markets, Basak and Shapiro (2001), Mansini et al. (2007) and Schied (2007) deal with risk management, portfolio choice and optimal investment, and Alexander et al. (2006) compare the minimization of the value at

\footnotetext{
1 Furthermore, Ogryczak and Ruszczynski (1999, 2002) point out that the variance is not compatible with the second order stochastic dominance if asymmetries are involved.
} 
risk $(\mathrm{VaR})$ and the conditional value at risk ( $\mathrm{CVaR})$ for a portfolio of derivatives.

The optimal reinsurance problem is a classical issue in Actuarial Science. Usually, authors consider the primary (or ceding) company viewpoint. A common approach attempts to minimize some measure of the first insurer risk after reinsurance, restricted to some premium condition. A first paper was by Borch (1960), who proved that the stop loss reinsurance minimizes the variance of the retained loss if premiums are calculated following the expected value principle. A few years later, Arrow (1963) also assumed the expected value principle and showed that the same stop loss reinsurance maximizes the expected utility of the terminal wealth of a risk-averse insurer.

The posterior research followed the ideas outlined in the articles above, trying to take into account more general risk measures and premium principles, which often give optimal contracts other than stop loss. In recent years, some interesting articles devoted to this subject have appeared. For example, Kaluszka (2001) still takes the variance of the retained loss as the risk function to be minimized, but he considers other premium principles such as the standard deviation principle and the variance principle. In addition, Gajec and Zagrodny (2004) consider more general symmetric and even asymmetric risk functions like the absolute deviation and the truncated variance of the retained loss, under the standard deviation premium principle. Young (1999) maximizes the expected utility of the final wealth under the distortion premium principle. ${ }^{2}$ Kaluszka (2005) studies reinsurance contracts with many convex premium principles (exponential, semi-deviation and semi-variance, Dutch, distortion, etc.). Other well known financial risk measures such as the VaR or the tail value at risk (TVaR) are also being considered. For example, Kaluszka (2005) uses the TVaR as a premium principle and Cai and Tan (2007) calculate the optimal retention for a stop loss reinsurance by considering the VaR and the conditional tail expectation risk measures (CTE), under the expected value premium principle. $^{3}$

This paper considers that the reinsurer's premium principle is given by a convex function, and deals with the optimal reinsurance problem if risk is measured by modern risk measures. ${ }^{4}$ These risk measures include every deviation measure, every expectation bounded risk measure, and most of the coherent, convex or consistent risk measures as particular cases. Thus it may be worth pointing out the level of generality of the analysis, since a unified approach is developed that essentially does not depend on the concrete risk measure to be used.

\footnotetext{
2 Further information about the "distortion premium principle" may be found in Denneberg (1994) or Wang (2000). The micro-economics literature studied an equivalent version in the 1980's.

3 The CVaR is also called TVaR, average value at risk (AVaR), expected shortfall (ES), CTE etc., although for some discrete random variables there might be some differences among the definitions used by several authors. The intuitive idea is that the CVaR represents the expectation of the random variable (agent final wealth) if it finally achieves values that are not higher than its VaR with the same level of confidence $\mu_{0}$. However, depending on the concrete definition of CVaR that one assumes, this interpretation may fail, mainly for discrete random variables.

In this paper we will consider the definition of CVaR given in Rockafellar et al. (2006). Thus, we have the equalities

$\operatorname{CVaR}_{\mu_{0}}(y)=\frac{1}{\mu_{0}} \int_{0}^{\mu_{0}} \operatorname{VaR}_{t}(y) \mathrm{d} t$

and

$\operatorname{CVaR}_{\mu_{0}}(y)=\operatorname{Max}\left\{-\mathbb{E}(y z) ; z \in L^{\infty}, 0 \leq z \leq 1 / \mu_{0}\right\}$

for every $y \in L^{1}, \mathbb{E}()$ denoting the mathematical expectation. With this definition, the CVaR is always coherent and expectation bounded (Rockafellar et al., 2006).

4 Insurance premiums are usually given by convex functions. See, for instance, Deprez and Gerber (1985).
}

The paper's outline is as follows. Section 2 will present the basic conditions and properties of the risk measure $\rho$ to be used. Section 3 provides our general optimal reinsurance problem. Since the risk measure is not differentiable in general, the resulting optimization problem is not differentiable either, and a significant part of this section will be devoted to overcoming this caveat. In fact, the results of this section will play a critical role in the rest of the article. We will use the representation theorem of risk measures of Rockafellar et al. (2006) so as to transform the initial optimal reinsurance problem into a minimax problem. Later, following an idea developed in Balbás and Romera (2007) and Balbás et al. (2009), the minimax problem is shown to be equivalent to a new differentiable and convex problem in Banach spaces. In particular, the dual variable belongs to the set of probabilities on the Borel $\sigma$-algebra of the sub-gradient of $\rho$. Since this fact would provoke a high degree of complexity when dealing with the optimality conditions of the new problem, Theorem 3 is one of the most important results in Section 3 and the whole article because it guarantees that the optimal dual solution will be a Dirac delta, and thus we can leave the use of general probability measures to characterizing the optimal reinsurance contract. The section ends by yielding necessary and sufficient optimality conditions. Theorem 4 may merit special interest, since it provides a variational principle that will often apply in the remaining sections.

Section 4 is devoted to verifying whether the usual types of reinsurance satisfy the optimality conditions, with special focus on quota share and stop loss contracts. Here, and in Section 5, we will assume that the reinsurer uses the expected value premium principle. Of course it is not necessary, since practical optimality conditions have been given in a much more general framework, but the specific solution of the optimization problem depends on the premium principle we take, and considering more than one would significantly enlarge the paper. As already indicated, previous literature measuring the insurer risk by a general risk measure is still scant. ${ }^{5}$ So it seems to be natural, and of interest, to analyze concrete problems by taking the most used premium principle.

It will be shown that a quota share reinsurance will barely be optimal, regardless of the risk function $\rho$, while a stop loss reinsurance much more easily satisfies the optimality conditions. The main reason is that the optimality of stop loss contracts is closely related to the existence of bang-bang-like solutions for the variational principle of Theorem 4.

Regardless of the level of generality of the analysis, it may be worthwhile studying particular risk functions in detail, and this is the focus of Section 5. So, the optimality conditions will be tested if $\rho$ equals the standard deviation, the absolute deviation and the conditional value at risk. Obviously, the optimality conditions may be tested in detail for many more alternative risk measures, but we had to make a choice. The standard deviation was selected because it has been very frequently used in Finance and Insurance, the absolute deviation has shown more adequate properties with respect to the stochastic dominance in presence of heavy tails and/or asymmetries (Ogryczak and Ruszczynski, 2002) and the conditional value at risk is becoming more and more important in Finance and Insurance because it also respects the stochastic dominance (Ogryczak and Ruszczynski, 2002), provides information about the degree of risk in monetary terms (capital requirements, reserves etc.), shows suitable analytic properties

\footnotetext{
5 Cai and Tan (2007) seems to be the first paper computing the optimal retention by considering the VaR and the CTE. However, these authors assume the expected value premium principle and only deal with stop loss reinsurance contracts, in the sense that another type of reinsurance is unfeasible.
} 
and is well-known and understood by many practitioners. For the three risk functions, we will find that the optimal strategy is closely related to a stop-loss-like reinsurance.

The last section of the paper points out the most important conclusions.

\section{Preliminaries and notations}

Consider the probability space $(\Omega, \mathcal{F}, \mu)$ composed of the set of "states of the world" $\Omega$, the $\sigma$-algebra $\mathcal{F}$ and the probability measure $\mu$. Consider also a couple of conjugate numbers $p \in$ $[1, \infty)$ and $q \in(1, \infty](i . e ., 1 / p+1 / q=1)$. As usual $L^{p}\left(L^{q}\right)$ denotes the Banach space of $\mathbb{R}$-valued random variables $y$ on $\Omega$ such that $\mathbb{E}\left(|y|^{p}\right)<\infty, \mathbb{E}()$ representing the mathematical expectation $\left(\mathbb{E}\left(|y|^{q}\right)<\infty\right.$, or $y$ essentially bounded if $\left.q=\infty\right)$. According to the Riesz Representation Theorem, we have that $L^{q}$ is the dual space of $L^{p}$.

Fix a future date $T$. Let

$\rho: L^{p} \longrightarrow \mathbb{R}$

be the general risk function that the insurer uses in order to control the risk of his final (at $T$ ) wealth. Denote by

$\Delta_{\rho}=\left\{z \in L^{q} ;-\mathbb{E}(y z) \leq \rho(y), \forall y \in L^{p}\right\}$.

The set $\Delta_{\rho}$ is obviously convex. We will assume that $\Delta_{\rho}$ is also $\sigma\left(L^{q}, L^{p}\right)$-compact and

$\rho(y)=\operatorname{Max}\left\{-\mathbb{E}(y z): z \in \Delta_{\rho}\right\}$

holds for every $y \in L^{p}$.

The set $\Delta_{\rho}$ is composed of those linear functions that are lower than the risk measure $\rho$. Following an interpretation of Artzner et al. (1999), amongst many others, every $z \in \Delta_{\rho}$ could be understood as a particular scenario, since the physical probability measure $\mu$ is distorted by $z$ in order to generate the $\sigma$-additive measure $v$ given by $\mathrm{d} v=z \mathrm{~d} \mu{ }^{6}$ Then $-\mathbb{E}(y z)$ would be a distorted expectation of $y$ under the scenario given by $z$. The minus symbol must arise if $y$ represents the wealth at $T$. It should be removed if $y$ represented capital losses. Equality (2) means that the risk is the maximum value of those distorted expectations of $y$ lower than $\rho$.

The assumptions above are quite natural. Indeed, they are closely related to the representation theorems of risk measures stated in Rockafellar et al. (2006), where the authors consider $p=2$. Following these authors' ideas, and bearing in mind the representation theorem 2.4.9 in Zalinescu (2002) for convex functions, it is easy to prove that the $\sigma\left(L^{q}, L^{p}\right)$-compactness of $\Delta_{\rho}$ and the fulfillment of (2) hold if:

(a) $\rho$ is a continuous expectation bounded risk measure in the sense of Rockafellar et al. (2006), ${ }^{7}$ in which case

$\Delta_{\rho} \subset\left\{z \in L^{q} ; \mathbb{E}(z)=1\right\}$

and $\rho(y) \geq-\mathbb{E}(y)$ for every $y \in L^{p}$ (with strict inequality if $y$ is not a constant or zero-variance random variable).

(b) $\rho$ is a continuous deviation (or deviation measure) in the sense of Rockafellar et al. (2006), ${ }^{8}$ in which case

$\Delta_{\rho} \subset\left\{z \in L^{q} ; \mathbb{E}(z)=0\right\}$

\footnotetext{
6 In fact, Artzner et al. (1999) used $\mu$-continuous finitely additive measures, rather than elements $z \in L^{q}$, i.e., they did not pay attention to the density function $z$ of $v$ with respect to $\mu$.

7 Furthermore, if $\rho$ is also coherent in the sense of Artzner et al. (1999) then

$\Delta_{\rho} \subset L_{+}^{q}=\left\{z \in L^{q} ; \mu(z \geq 0)=1\right\}$.

8 Moreover, according to Theorem 2.2.20 in Zalinescu (2002), $\rho$ is continuous if and only if $\rho$ is lower semi-continuous. The same equivalence holds if $\rho$ is expectation bounded risk measure.
}

and $\rho(y) \geq 0$ for every $y \in L^{p}$ (with strict inequality if $y$ is not a constant or zero-variance random variable).

Particular interesting examples are the conditional value at risk (CVaR) of Rockafellar et al. (2006), the dual power transform (DPT) of Wang (2000), the Wang measure (Wang, 2000), the $p$-deviation given by $\rho(y)=\left[\mathbb{E}\left(|\mathbb{E}(y)-y|^{p}\right)\right]^{1 / p}$, or the downside $p$-semideviation given by $\rho(y)=\left[\mathbb{E}\left(|\operatorname{Max}\{\mathbb{E}(y)-y, 0\}|^{p}\right)\right]^{1 / p}$, amongst many others.

Assumption 1. Henceforth we will assume that $\Delta_{\rho}$ is $\sigma\left(L^{q}, L^{p}\right)$ compact, (2) holds and $\mathbb{E}()$ remains constant on $\Delta_{\rho}$. Denoting $\mathbb{E}(z)=\tilde{E} \in \mathbb{R}$ for every $z \in \Delta_{\rho}$ we will also suppose that $\tilde{E} \geq 0$ and

$\rho(y) \geq-\mathbb{E}(y) \tilde{E}$

holds for every $y \in L^{p}$.

Proposition 1. Under Assumption 1 the constant random variable $z=\tilde{E}$ a.s. belongs to $\Delta_{\rho}$.

Proof. It immediately follows from (1) and (4).

\section{Optimal reinsurance: Primal and dual problems and opti- mality conditions}

Consider that the insurance company receives the fixed amount $S_{0}$ (premium) and will have to pay the random variable

$y_{0} \in L_{+}^{p}=\left\{y \in L^{p}: \mu(y \geq 0)=1\right\}$

within a given period $[0, T]$ (claims). Suppose also that a reinsurance contract is signed in such a way that the company will only pay $y \in L^{p}$, whereas the reinsurer will pay $y_{0}-y$. If the reinsurer premium principle is given by the continuous convex function,

$\pi: L^{p} \longrightarrow \mathbb{R}$

such that $\pi(0)=0$, and $S_{1}>0$ is the highest amount that the insurer would like to pay for the contract, then the insurance company will choose $y$ (optimal retention) so as to solve

$\left\{\begin{array}{l}\operatorname{Min} \rho\left(S_{0}-y-\pi\left(y_{0}-y\right)\right) \\ \pi\left(y_{0}-y\right) \leq S_{1} \\ 0 \leq y \leq y_{0}\end{array}\right.$

Conditions $\pi(0)=0$ and $S_{1}>0$ imply that $y=y_{0}$ satisfies the constraint, so (5) is never unfeasible.

In general $\rho$ will be non-differentiable and therefore so will be Problem (5). To overcome this caveat we will follow the method proposed in Balbás et al. (2009). So, bearing in mind (2), Problem (5) is equivalent to Problem

$\left\{\begin{array}{l}\operatorname{Min} \theta \\ \theta+\mathbb{E}\left(\left(S_{0}-y-\pi\left(y_{0}-y\right)\right) z\right) \geq 0, \quad \forall z \in \Delta_{\rho} \\ \pi\left(y_{0}-y\right) \leq S_{1} \\ \theta \in \mathbb{R}, 0 \leq y \leq y_{0}\end{array}\right.$

in the sense that $y$ solves (5) if and only if there exists $\theta \in \mathbb{R}$ such that $(\theta, y)$ solves $(6)$, in which case

$\theta=\rho\left(S_{0}-y-\pi\left(y_{0}-y\right)\right)$

holds. Since Assumption 1 implies that

$\mathbb{E}\left(\left(S_{0}-y-\pi\left(y_{0}-y\right)\right) z\right)=\left(S_{0}-\pi\left(y_{0}-y\right)\right) \tilde{E}-\mathbb{E}(y z)$

(5) and (6) are equivalent to

$\left\{\begin{array}{l}\operatorname{Min} \theta \\ \theta+\left(S_{0}-\pi\left(y_{0}-y\right)\right) \tilde{E}-\mathbb{E}(y z) \geq 0, \quad \forall z \in \Delta_{\rho} \\ \pi\left(y_{0}-y\right) \leq S_{1} \\ \theta \in \mathbb{R}, 0 \leq y \leq y_{0}\end{array}\right.$ 
The objective function of (7) is obviously differentiable (and linear) so the major caveat of (5) has been overcome. Suppose that (7) is a convex problem. ${ }^{9}$ Its first constraint is valued on the Banach space $\mathcal{C}\left(\Delta_{\rho}\right)$ of real-valued and continuous functions on the $\left(w e a k^{*}\right)$ compact space $\Delta_{\rho}$. Following Luenberger (1969), since the dual of $\mathcal{C}\left(\Delta_{\rho}\right)$ is $\mathcal{M}\left(\Delta_{\rho}\right),{ }^{10}$ the space of inner regular real valued $\sigma$ additive measures on the Borel $\sigma$-algebra of $\Delta_{\rho}$ (endowed with the weak* topology), the Lagrangian function

$\mathcal{L}: \mathbb{R} \times L^{p} \times \mathbb{R} \times \mathcal{M}\left(\Delta_{\rho}\right) \longrightarrow \mathbb{R}$

becomes

$$
\begin{aligned}
& \mathcal{L}(\theta, y, \tau, v)= \\
& \quad \theta\left(1-\int_{\Delta_{\rho}} \mathrm{d} v(z)\right)-\left(S_{0}-\pi\left(y_{0}-y\right)\right) \tilde{E} \int_{\Delta_{\rho}} \mathrm{d} v(z) \\
& +\int_{\Delta_{\rho}} \mathbb{E}(y z) \mathrm{d} v(z)+\tau\left(\pi\left(y_{0}-y\right)-S_{1}\right) .
\end{aligned}
$$

The element $(\tau, v) \in \mathbb{R} \times \mathcal{M}\left(\Delta_{\rho}\right)$ is dual feasible if and only if it belongs to the non-negative cone $\mathbb{R}_{+} \times \mathcal{M}_{+}\left(\Delta_{\rho}\right)$ and

$\operatorname{Inf}\left\{\mathcal{L}(\theta, y, v, \tau): \theta \in \mathbb{R}, 0 \leq y \leq y_{0}\right\}>-\infty$,

in which case the infimum above equals the dual objective on $(\tau, v)$. Hence, the dual problem of $(7)$ becomes

$$
\left\{\begin{array}{l}
\operatorname{Max}\left(-S_{0} \tilde{E}-\tau S_{1}+\operatorname{Inf}_{0 \leq y \leq y_{0}}\left\{\int_{\Delta_{\rho}} \mathbb{E}(y z) \mathrm{d} v(z)\right.\right. \\
\left.\left.+(\tilde{E}+\tau) \pi\left(y_{0}-y\right)\right\}\right) \quad \tau \in \mathbb{R}_{+}, v \in \mathcal{P}\left(\Delta_{\rho}\right)
\end{array}\right.
$$

$\mathcal{P}\left(\Delta_{\rho}\right)$ denoting the set composed of those elements in $\mathcal{M}\left(\Delta_{\rho}\right)$ that are probabilities.

$\mathcal{P}\left(\Delta_{\rho}\right)$ is convex, and the Alaoglu Theorem easily leads to the compactness of $\mathcal{P}\left(\Delta_{\rho}\right)$ when endowed with the $\sigma\left(\mathcal{M}\left(\Delta_{\rho}\right)\right.$, $\mathcal{C}\left(\Delta_{\rho}\right)$ )-topology (Horvàth, 1966). In addition, given $z \in \Delta_{\rho}$ we will denote by $\delta_{z} \in \mathcal{P}\left(\Delta_{\rho}\right)$ the usual Dirac delta that concentrates the mass on $\{z\}$, i.e., $\delta_{z}(\{z\})=1$ and $\delta_{z}\left(\Delta_{\rho} \backslash\{z\}\right)=0$. It is known that the set of extreme points of $\mathcal{P}\left(\Delta_{\rho}\right)$ is given by

$\operatorname{ext}\left(\mathcal{P}\left(\Delta_{\rho}\right)\right)=\left\{\delta_{z} ; z \in \Delta_{\rho}\right\}$,

though we will not have to draw on this result. The objective function in (8) is linear which, along with (9), suggests that the solution of (8) could be achieved in $\left\{\delta_{z} ; z \in \Delta_{\rho}\right\}$. Let us show that this guesstimate is correct.

Lemma 2 (Mean Value Theorem). Let $v \in \mathcal{P}\left(\Delta_{\rho}\right)$. Then there exists $z_{v} \in \Delta_{\rho}$ such that

$$
\int_{\Delta_{\rho}} \mathbb{E}(y z) \mathrm{d} v(z)=\mathbb{E}\left(y z_{v}\right)
$$

holds for every $y \in L^{p}$.

\footnotetext{
9 Due to (3), Problem (7) is convex if $\rho$ is coherent. There are many other cases leading to a convex optimization problem. For instance, if the reinsurer uses the expected value premium principle then ( 7 ) is convex regardless of the coherence of $\rho$.

If (7) were not convex then an alternative analysis might be implemented by assuming that $\pi$ is differentiable and using those properties of differentiable mathematical programming in Banach spaces (Luenberger, 1969), but this study is beyond our scope.

10 See also Horvàth (1966).
}

Proof. Consider the linear function

$L^{p} \ni y \longrightarrow \varphi(y)=\int_{\Delta_{\rho}} \mathbb{E}(y z) \mathrm{d} v(z) \in \mathbb{R}$.

$\varphi$ is clearly continuous because for every sequence $\left(y_{n}\right)_{n=1}^{\infty}$ converging to zero in $L^{p}$ the sequence of functions

$L^{q} \ni z \longrightarrow \mathbb{E}\left(y_{n} z\right) \in \mathbb{R}$

uniformly converges to zero on bounded sets of $L^{q}$, and, consequently,

$\left(\int_{\Delta_{\rho}} \mathbb{E}\left(y_{n} z\right) \mathrm{d} v(z)\right)_{n=1}^{\infty}$

converges to zero if $\Delta_{\rho}$ is bounded. $\Delta_{\rho}$ is bounded because it is $\sigma\left(L^{q}, L^{p}\right)$-compact.

Since $\varphi$ is linear and continuous, the Riesz Representation Theorem guarantees the existence of $z_{v} \in L^{q}$ such that (10) holds. Thus, it only remains to show that $z_{v} \in \Delta_{\rho}$, i.e., according to (1), we must prove the inequality

$-\mathbb{E}\left(y z_{v}\right) \leq \rho(y), \quad \forall y \in L^{p}$.

(10) points out that it is sufficient to see

$-\int_{\Delta_{\rho}} \mathbb{E}(y z) \mathrm{d} v(z) \leq \rho(y), \quad \forall y \in L^{p}$.

For every $z \in \Delta_{\rho}$ one has that $-\mathbb{E}(y z) \leq \rho(y)$ for every $y \in L^{p}$, and, therefore,

$-\int_{\Delta_{\rho}} \mathbb{E}(y z) \mathrm{d} v(z) \leq \int_{\Delta_{\rho}} \rho(y) \mathrm{d} v(z)=\rho(y)$,

for every $y \in L^{p}$.

Theorem 3. If $(\tau, v) \in \mathbb{R}_{+} \times \mathcal{P}\left(\Delta_{\rho}\right)$ solves (8) then there exists $z \in \Delta_{\rho}$ such that $\left(\tau, \delta_{z_{v}}\right)$ solves (8).

Proof. Consider ( $v, \tau)$ solving (8) and take $z_{v} \in \Delta_{\rho}$ satisfying (10). Then, for every $y \in L_{+}^{p}$ we have that

$$
\begin{aligned}
& =\operatorname{Inf}_{0 \leq y \leq y_{0}}\left\{\int_{\Delta_{\rho}} \mathbb{E}(y z) \mathrm{d} v(z)+(\tilde{E}+\tau) \pi\left(y_{0}-y\right)\right\} \\
& =\operatorname{Inf}_{0 \leq y_{0}}\left\{\mathbb{E}\left(y z_{\nu}\right)+(\tilde{E}+\tau) \pi\left(y_{0}-y\right)\right\} .
\end{aligned}
$$

Thus the result trivially follows because the objective values of (8) in $(\tau, v)$ and $\left(\tau, \delta_{z_{v}}\right)$ are identical.

Remark 1. The latter theorem leads to significant consequences. In particular, we can consider the alternative and far simpler dual problem

$$
\begin{aligned}
& \operatorname{Max}\left(-S_{0} \tilde{E}-\tau S_{1}+\operatorname{Inf}_{0 \leq y \leq y_{0}}\left\{\mathbb{E}(y z)+(\tilde{E}+\tau) \pi\left(y_{0}-y\right)\right\}\right) \\
& \tau \in \mathbb{R}_{+}, z \in \Delta_{\rho}
\end{aligned}
$$

where $z \in \Delta_{\rho}$ plays the role of $v \in \mathcal{P}\left(\Delta_{\rho}\right)$. Indeed, notice that Theorem 3 guarantees that we only have to focus on the (8)feasible solutions taking the form $\left(\tau, \delta_{z}\right)$ for some $z \in \Delta_{\rho}$, and for such a feasible solution (8) is equivalent to (11).

Bearing in mind the latter remark, the Karush-Kuhn-Tucker conditions of (7) may be given by using (11) rather than (8). Then 
they become (Luenberger, 1969)

$$
\left\{\begin{array}{l}
\theta^{*}+\left(S_{0}-\pi\left(y_{0}-y^{*}\right)\right) \tilde{E}-\mathbb{E}\left(y^{*} z^{*}\right)=0 \\
\theta^{*}+\left(S_{0}-\pi\left(y_{0}-y^{*}\right)\right) \tilde{E}-\mathbb{E}\left(y^{*} z\right) \geq 0, \quad \forall z \in \Delta_{\rho} \\
\tau^{*}\left(\pi\left(y_{0}-y^{*}\right)-S_{1}\right)=0 \\
\pi\left(y_{0}-y^{*}\right)-S_{1} \leq 0 \\
\mathbb{E}\left(y^{*} z^{*}\right)+\left(\tilde{E}+\tau^{*}\right) \pi\left(y_{0}-y^{*}\right) \leq \mathbb{E}\left(y z^{*}\right) \\
\quad+\left(\tilde{E}+\tau^{*}\right) \pi\left(y_{0}-y\right), \quad \forall 0 \leq y \leq y_{0} \\
\theta^{*} \in \mathbb{R}, 0 \leq y^{*} \leq y_{0}, \tau^{*} \geq 0, z^{*} \in \Delta_{\rho}
\end{array}\right.
$$

and they are necessary and sufficient optimality conditions to guarantee that $\left(\theta^{*}, y^{*}\right)$ solves (7) and $\left(\tau^{*}, z^{*}\right)$ solves (11). We are dealing with infinite dimensional Banach spaces and the socalled "duality gap" between (7) and (11) might arise. We must see that this pathological situation does not apply here, in order to guarantee that (12) really characterizes primal and dual solutions (see Luenberger, 1969). ${ }^{11}$ It is sufficient to show that the Slater qualification holds, i.e., the existence of a primal feasible solution $(\theta, y)$ satisfying the two primal constraints as strict inequalities. Being $\Delta_{\rho}$ compact, one can take $y=y_{0}$ and

$\theta>\operatorname{Max}\left\{\mathbb{E}\left(y_{0} z\right)-S_{0} \tilde{E}, z \in \Delta_{\rho}\right\}$.

On the other hand, the fulfillment of the Slater qualification also ensures that (11) is solvable, i.e., it attains its optimal value.

Finally, bearing in mind the equivalence between (5) and (7), it may be interesting to recall that the solution $z^{*}$ provides us with the sensitivity of the optimal risk level

$\rho\left(S_{0}-y^{*}-\pi\left(y_{0}-y^{*}\right)\right)$

with respect to the initial data $S_{0}$ and $y_{0}$, while $\tau^{*}$ measures sensitivity with respect to $S_{1}$.

Let us end this section with alternative optimality conditions implied by (12) that will often apply throughout the rest of the paper.

Theorem 4 (Variational Principle). Suppose that $0 \leq y^{*} \leq y_{0}$ in $L^{p}$. $y^{*}$ solves (5) if and only if there exist $\tau^{*} \in \mathbb{R}_{+}$and $z^{*} \in \Delta_{\rho}$ such that

$$
\begin{cases}\mathbb{E}\left(y^{*} z^{*}\right) \geq \mathbb{E}\left(y^{*} z\right), & \forall z \in \Delta_{\rho} \\ \mathbb{E}\left(y^{*} z^{*}\right)+\left(\tilde{E}+\tau^{*}\right) \pi\left(y_{0}-y^{*}\right) & \\ \quad \leq \mathbb{E}\left(y z^{*}\right)+\left(\tilde{E}+\tau^{*}\right) \pi\left(y_{0}-y\right), & \forall 0 \leq y \leq y_{0} \\ \pi\left(y_{0}-y^{*}\right)-S_{1} \leq 0 & \\ \tau^{*}\left(\pi\left(y_{0}-y^{*}\right)-S_{1}\right)=0 . & \end{cases}
$$

In such a case $\theta^{*}=\mathbb{E}\left(y^{*} z^{*}\right)-\left(S_{0}-\pi\left(y_{0}-y^{*}\right)\right) \tilde{E}, y^{*}, \tau^{*}$ and $z^{*}$ solve both (7) and (11) respectively.

Proof. Suppose that $y^{*}$ solves (5), and take a primal solution $\left(\theta^{*}, y^{*}\right)$ and a dual one $\left(\tau^{*}, z^{*}\right)$. The first and second conditions in (12) trivially lead to the first one in (13), and the remaining equations in (13) trivially follow from (12).

Conversely, if $y^{*}$ satisfies (13) then it may be immediately verified that the proposed solution $\left(\theta^{*}, y^{*}, \tau^{*}, z^{*}\right)$ satisfies (12).

Remark 2. Conditions (12) are necessary and sufficient and therefore they are a quite useful tool. Nevertheless, in practical examples and applications it might be difficult to find an explicit solution of the system generated by (12). Things become much

\footnotetext{
11 The existence of duality gaps and the lack of Lagrange or Karush-Kuhn-Tucker multipliers is not so unusual in actuarial or financial problems. See, for instance, Jin et al. (2008) for noteworthy counter-examples in portfolio selection.
}

easier if we are able to compute the solution of (7) or (11) by an alternative algorithm, since then (12) easily applies to solve the remaining problem. This is, for instance, the way followed in Balbás and Romera (2007) or Balbás et al. (2009), where the authors deal with infinite-dimensional linear programming in order to solve risk minimization problems associated with usual financial topics. Following the ideas of these authors, we could try to develop an algorithm so as to solve (11) under appropriate assumptions, and then we could use (12) so as to solve (7). However, in this paper we will prefer to draw on the variational principle provided by the condition $\mathbb{E}\left(y^{*} z^{*}\right) \geq \mathbb{E}\left(y^{*} z\right)$ of Theorem 4 . Indeed, the next two sections will show that it may be very useful in both theoretical approaches and practical situations. In particular, when dealing with practical applications, it may yield an interesting relationship between the solution $y^{*}$ of (7) and the solution $z^{*}$ of (11), that may be found by solving the simple and frequently linear problem

$\left\{\begin{array}{l}\operatorname{Max} \mathbb{E}\left(y^{*} z\right) \\ z \in \Delta_{\rho}\end{array}\right.$

Some illustrative examples will be studied in Section 5 .

\section{Particular reinsurance contracts}

This section will be devoted to verifying whether the most important (or usual) reinsurance contracts solve Systems (12) or (13). In particular, we will focus on quota share and stop loss reinsurance contracts. Despite the level generality of the previous analyses, the solutions of (12) or (13) will depend on the specific assumptions one imposes. Henceforth we will assume that the reinsurer uses the expected value premium principle. As indicated in the introduction, previous literature considering a general risk measure is scant, so it seems to be natural and of interest to analyze concrete problems by taking the most used premium principle. Nevertheless, it is worth pointing out that the developments of the previous section are much more general, and therefore they also apply to alternative premium principles.

The assumption $\mu\left(y_{0}>0\right)=1$ seems to be totally compatible with the empirical evidence and non restrictive in practice, since it only indicates that the existence of claims is guaranteed. From now on we will impose this assumption. Summarizing the new hypotheses of this section, we have:

Assumption 2. Henceforth, we will assume that the reinsurer applies the expected value premium principle, and the equality $\mu\left(y_{0}>0\right)=1$ holds.

Accordingly, there exists $k \geq 1$ such that

$\pi(y)=k \mathbb{E}(y)$

for every $y \in L^{p}$. Consequently, the necessary and sufficient optimality conditions (13) become

$$
\left\{\begin{array}{l}
\mathbb{E}\left(y^{*} z^{*}\right) \geq \mathbb{E}\left(y^{*} z\right), \quad \forall z \in \Delta_{\rho} \\
\mathbb{E}\left(y^{*} z^{*}\right)-\left(\tilde{E}+\tau^{*}\right) k \mathbb{E}\left(y^{*}\right) \\
\quad \leq \mathbb{E}\left(y z^{*}\right)-\left(\tilde{E}+\tau^{*}\right) k \mathbb{E}(y), \quad \forall 0 \leq y \leq y_{0} \\
k \mathbb{E}\left(y_{0}-y^{*}\right)-S_{1} \leq 0 \\
\tau^{*}\left(k \mathbb{E}\left(y_{0}-y^{*}\right)-S_{1}\right)=0
\end{array}\right.
$$

in which case the optimal value of $\theta$ is

$$
\theta^{*}=\mathbb{E}\left(y^{*} z^{*}\right)-\left(S_{0}-k \mathbb{E}\left(y_{0}-y^{*}\right)\right) \tilde{E} .
$$

Next let us present a simple lemma simplifying the second condition of (15). 
Lemma 5. Let $z^{*} \in L^{q}, y^{*} \in L^{p}$ with $0 \leq y^{*} \leq y_{0}$, and $\tau^{*} \in \mathbb{R}$. Then, $\mathbb{E}\left(y^{*} z^{*}\right)-\left(\tilde{E}+\tau^{*}\right) k \mathbb{E}\left(y^{*}\right) \leq \mathbb{E}\left(y z^{*}\right)-\left(\tilde{E}+\tau^{*}\right) k \mathbb{E}(y)$

holds for every $y \in L^{p}$ with $0 \leq y \leq y_{0}$ if and only if there exists a measurable partition

$\Omega=\Omega_{1} \cup \Omega_{2} \cup \Omega_{3}$

such that

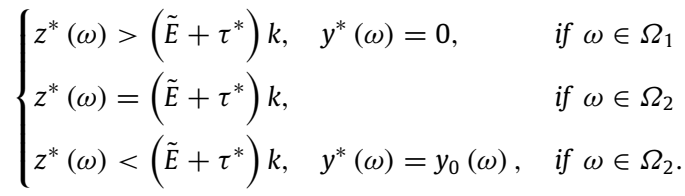

Proof. It is obvious if we realize that the solution of

$$
\left\{\begin{array}{l}
\operatorname{Min} \mathbb{E}\left(y\left(z^{*}-\left(\tilde{E}+\tau^{*}\right) k\right)\right) \\
0 \leq y \leq y_{0}
\end{array}\right.
$$

must be as large as possible (i.e., must equal $y_{0}$ ) whenever $z^{*}-$ $\left(\tilde{E}+\tau^{*}\right) k<0$ and as small as possible (i.e., zero) if $z^{*}-$ $\left(\tilde{E}+\tau^{*}\right) k>0$, whereas the value of this solution is not relevant at all if $z^{*}-\left(\tilde{E}+\tau^{*}\right) k=0$.

Next let us focus on quota-share-like reinsurance contracts. Obviously, $y \in L^{p}$ is said to be a quota share reinsurance if there exists $\alpha \in[0,1]$ such that $y=\alpha y_{0}$.

Theorem 6. (a) Suppose that $\tilde{E}>0 . y^{*}=\alpha y_{0}$ with $\alpha \in[0,1)$ is optimal if and only if $\alpha \geq 1-\frac{s_{1}}{\mathbb{E}\left(y_{0}\right)}, k=1$ and $\alpha \mathbb{E}\left(y_{0} z\right) \leq \alpha \tilde{E} \mathbb{E}\left(y_{0}\right)$ for every $z \in \Delta_{\rho}$. In such a case $\theta^{*}=\left((1-\alpha) \mathbb{E}\left(y_{0}\right)-S_{0}\right) \tilde{E}$, $y^{*}=\alpha y_{0}, \tau^{*}=0$ and $z^{*}=\tilde{E}$ solve the primal and the dual problem respectively. ${ }^{12}$

(b) Suppose that $\tilde{E}=0 . y^{*}=\alpha y_{0}$ with $\alpha \in[0,1)$ is optimal if and only if $\alpha \geq 1-\frac{s_{1}}{k \mathbb{E}\left(y_{0}\right)}$ and $\alpha \mathbb{E}\left(y_{0} z\right) \leq 0$ for every $z \in \Delta_{\rho}$. In such a case $\theta^{*}=0, y^{*}=\alpha y_{0}, \tau^{*}=0$ and $z^{*}=0$ solve the primal and the dual problem respectively.

(c) If $\rho$ is a deviation measure then $y^{*}=\alpha y_{0}$ with $\alpha \in(0,1)$ is optimal if and only if $\alpha \geq 1-\frac{s_{1}}{k \mathbb{E}\left(y_{0}\right)}$ and $y_{0}$ is constant (zerovariance).

Proof. Suppose that $y^{*}=\alpha y_{0}$ with $\alpha \in[0,1)$ is the optimal retention. Then (15) and (17) lead to the existence of a partition of $\Omega$, and $\tau^{*} \geq 0$ and $z^{*} \in \Delta_{\rho}$ such that

$$
\begin{cases}\alpha \mathbb{E}\left(y_{0} z^{*}\right) \geq \alpha \mathbb{E}\left(y_{0} z\right), \quad \forall z \in \Delta_{\rho} & \\ z^{*}>\left(\tilde{E}+\tau^{*}\right) k, \quad \alpha y_{0}=0, & \text { in } \Omega_{1} \\ z^{*}=\left(\tilde{E}+\tau^{*}\right) k, & \text { in } \Omega_{2} \\ z^{*}<\left(\tilde{E}+\tau^{*}\right) k, \quad \alpha y_{0}=y_{0}, & \text { in } \Omega_{3} \\ k(1-\alpha) \mathbb{E}\left(y_{0}\right) \leq S_{1} \\ \tau^{*}\left(k(1-\alpha) \mathbb{E}\left(y_{0}\right)-S_{1}\right)=0\end{cases}
$$

The fourth condition would imply $\alpha=1$, so $\Omega_{3}$ must be a null set that can be considered void. Therefore $z^{*} \geq\left(\tilde{E}+\tau^{*}\right) k$ and taking

\footnotetext{
12 Recall that $z^{*}=\tilde{E} \in \Delta_{\rho}$ owing to Proposition 1 . Recall also the $\tilde{E}=1$ for expectation bounded risk measures and $\tilde{E}=0$ for deviation measures.
}

expectations $\tilde{E} \geq\left(\tilde{E}+\tau^{*}\right) k$, which leads to $\tau^{*} \leq \frac{\tilde{E}(1-k)}{k} \leq 0$. Since the opposite inequality must hold we have $\tau^{*}=0$,

$\tilde{E} \geq \tilde{E} k$

and

$z^{*} \geq \tilde{E} k$.

(a) Suppose that $\tilde{E}>0$. Then $k \leq 1$ follows from (19) and the opposite inequality is given by the assumptions on $k$. $\alpha \geq$ $1-\frac{S_{1}}{E\left(y_{0}\right)}$ follows from the fifth expression in (18). (20) implies that $z^{*} \geq \tilde{E}$, and thus $z^{*}=\tilde{E}$ owing to $\mathbb{E}\left(z^{*}\right)=\tilde{E}$. Then $\alpha \mathbb{E}\left(y_{0} z\right) \leq \alpha \tilde{E} \mathbb{E}\left(y_{0}\right)$ is implied by the first condition in (18), and $\theta^{*}=\left((1-\alpha) \mathbb{E}\left(y_{0}\right)-S_{0}\right) \tilde{E}$ follows from (16).

Conversely, if the given conditions hold, then it is easy to verify that the proposed solution satisfies (15) and (17).

(b) Suppose that $\tilde{E}=0$. Then the given conditions may be proved by following similar arguments as in the proof above. Furthermore, the converse implication may be proved with similar arguments as well.

(c) Suppose that $\rho$ is a deviation measure and $y^{*}=\alpha y_{0}$ with $\alpha \in(0,1)$ is optimal. Then (b) shows that $\theta^{*}=0$ is the optimal value of (7), and therefore the optimal value of (5). Thus

$0=\rho\left(S_{0}-\alpha y_{0}-k(1-\alpha) \mathbb{E}\left(y_{0}\right)\right)=\alpha \rho\left(-y_{0}\right)$

which implies that $-y_{0}$ is constant and, thus, so is $y_{0}$.

The converse is obvious because every deviation measure vanishes on zero-variance random variables and is positive for non-constant ones.

Remark 3. Theorem 6 has clarified that quota share contracts will never be optimal in practice. Indeed, for expectation bounded risk measures we would need $k=1$, which will not hold. For deviations $y_{0}$ should be zero-variance, which will be even more improbable.

Next let us verify stop-loss-like reinsurance contracts. Obviously, $y \in L^{p}$ and lying between 0 and $y_{0}$ is said to be a stop loss reinsurance if there exists $\alpha>0$ such that

$y= \begin{cases}y_{0}, & y_{0} \leq \alpha \\ \alpha & \text { otherwise. }\end{cases}$

Hereafter the random variable of (21) will be denoted by $y_{0}^{\alpha}$. Without loss of generality we will assume in the remainder of this section that $\mu\left(y_{0}>\alpha\right)>0$, i.e., $y_{0}^{\alpha} \neq y_{0}$.

Theorem 7. Suppose that $\mu\left(y_{0}>\alpha\right)>0$ and $k \mathbb{E}\left(y_{0}-y_{0}^{\alpha}\right)<S_{1}$ hold. Denote $\Omega_{\alpha}=\left\{\omega \in \Omega ; y_{0}(\omega)>\alpha\right\}$. Then:

(a) $y_{0}^{\alpha}$ solves (5) if and only if there exists $z^{*} \in \Delta_{\rho}$ such that

$z^{*} \leq k \tilde{E}$,

$z^{*}(\omega)=k \tilde{E}, \quad \omega \in \Omega_{\alpha}$

and

$\mathbb{E}\left(y_{0}^{\alpha} z^{*}\right) \geq \mathbb{E}\left(y_{0}^{\alpha} z\right)$

for every $z \in \Delta_{\rho}$. In such a case $\theta^{*}=\mathbb{E}\left(y_{0}^{\alpha} z^{*}\right)-$ $\left(S_{0}-k \mathbb{E}\left(y_{0}-y_{0}^{\alpha}\right)\right) \tilde{E}, y_{0}^{\alpha}, \tau^{*}=0$ and $z^{*}$ solve both the primal and the dual problem respectively.

(b) Suppose that $\tilde{E}=0 . y_{0}^{\alpha}$ solves (5) if and only if

$\mathbb{E}\left(y_{0}^{\alpha} z\right) \leq 0$

for every $z \in \Delta_{\rho}$. In such a case $\theta^{*}=0, y_{0}^{\alpha}, z^{*}=0$ and $\tau^{*}=0$ solve both the primal and the dual problem.

(c) Suppose that $\rho$ is a deviation measure. Then $y_{0}^{\alpha}$ solves (5) if and only if $y_{0}^{\alpha}$ is constant (zero-variance). 
Proof. Suppose that $y_{0}^{\alpha}$ solves (5). Then (15) and (17) give $\tau^{*}=0$ and

$$
\begin{cases}\mathbb{E}\left(y_{0}^{\alpha} z^{*}\right) \geq \mathbb{E}\left(y_{0}^{\alpha} z\right), \quad \forall z \in \Delta_{\rho} & \\ z^{*}>\tilde{E} k, \quad y_{0}^{\alpha}=0, & \text { in } \Omega_{1} \\ z^{*}=\tilde{E} k, & \text { in } \Omega_{2} \\ z^{*}<\tilde{E} k, \quad y_{0}^{\alpha}=y_{0}, & \text { in } \Omega_{3} .\end{cases}
$$

The set $\Omega_{1}$ may be considered empty (or null), since $y_{0}^{\alpha}=0$ cannot hold due to $\mu\left(y_{0}>0\right)=1$. Thus (22) is fulfilled. In addition, $\Omega_{3}$ must be included in the complementary of $\Omega_{\alpha}$, since $y_{0}^{\alpha}=y_{0}$ implies $y_{0} \leq \alpha$. Hence $\Omega_{\alpha}$ is included in $\Omega_{2}$ and (23) holds. Finally, (24) trivially follows from the first condition in (26) and

$$
\theta^{*}=\mathbb{E}\left(y_{0}^{\alpha} z^{*}\right)-\left(S_{0}-k \mathbb{E}\left(y_{0}-y_{0}^{\alpha}\right)\right) \tilde{E}
$$

is a consequence of $(16)$.

(a) It only remains to prove the converse implication, which is trivial since one only has to verify that the proposed solution satisfies (15) and (17).

(b) According to (22) $z^{*} \leq 0$, which implies $z^{*}=0$ because $E\left(z^{*}\right)=0\left(z^{*} \in \Delta_{\rho}\right)$. Hence, (25) follows from (24), and the converse is immediate since one only needs to check the proposed solution in (15).

(c) If $\rho$ is a deviation measure then (b) shows that $\theta^{*}=0$ is the optimal value of (7), and therefore the optimal value of (5). Thus

$0=\rho\left(S_{0}-y_{0}^{\alpha}-k \mathbb{E}\left(y_{0}-y_{0}^{\alpha}\right)\right)=\rho\left(-y_{0}^{\alpha}\right)$

which implies that $-y_{0}^{\alpha}$ is constant, and thus so is $y_{0}^{\alpha}$. The converse is obvious because every deviation measure vanishes on zerovariance random variables and is positive for non-constant ones.

If we remove the assumption $k \mathbb{E}\left(y_{0}-y_{0}^{\alpha}\right)<S_{1}$ then things become a little bit more complex.

Theorem 8. Suppose that $\mu\left(y_{0}>\alpha\right)>0$ and $k \mathbb{E}\left(y_{0}-y_{0}^{\alpha}\right)=S_{1}$ hold. Denote $\Omega_{\alpha}=\left\{\omega \in \Omega ; y_{0}(\omega)>\alpha\right\}$. Then, $y_{0}^{\alpha}$ solves (5) if and only if there exist $z^{*} \in \Delta_{\rho}$ and $\tau^{*} \in \mathbb{R}_{+}$such that

$z^{*} \leq k \tilde{E}+\tau^{*}$,

$z^{*}(\omega)=k \tilde{E}+\tau^{*}, \quad \omega \in \Omega_{\alpha}$

and

$E\left(y_{0}^{\alpha} z^{*}\right) \geq E\left(y_{0}^{\alpha} z\right)$

for every $z \in \Delta_{\rho}$. In such a case $\theta^{*}=\mathbb{E}\left(y_{0}^{\alpha} z^{*}\right)-$ $\left(S_{0}-k \mathbb{E}\left(y_{0}-y_{0}^{\alpha}\right)\right) \tilde{E}, y_{0}^{\alpha}, \tau^{*}$ and $z^{*}$ solve both the primal and the dual problem respectively.

We will not give any proof of this result because it is absolutely analogous to the proof of the previous theorem.

Remark 4. Despite the fact that Theorem 7 seems to be more exhaustive than Theorem 8 , condition $k \mathbb{E}\left(y_{0}-y_{0}^{\alpha}\right)<S_{1}$ is "more ambiguous" than $k \mathbb{E}\left(y_{0}-y_{0}^{\alpha}\right)=S_{1}$. Indeed, there cannot be two different values of $\alpha$ satisfying the equality because if $\alpha_{1}<\alpha_{2}$, $\mu\left(y_{0}>\alpha_{2}\right)>0$ and $\mathbb{E}\left(y_{0}^{\alpha_{1}}\right)=\mathbb{E}\left(y_{0}^{\alpha_{2}}\right)$ then $\mathbb{E}\left(y_{0}^{\alpha_{2}}-y_{0}^{\alpha_{1}}\right)=0$ and $y_{0}^{\alpha_{2}}-y_{0}^{\alpha_{1}} \geq 0$ imply $y_{0}^{\alpha_{2}}-y_{0}^{\alpha_{1}}=0$. Whence,

$y_{0}^{\alpha_{1}}(\omega)=\alpha_{1}<\alpha_{2}=y_{0}^{\alpha_{2}}(\omega)$

for $\omega \in \Omega_{\alpha_{2}}$ implies $\mu\left(y_{0}>\alpha_{2}\right)=0$, against the assumptions.

Consequently, it is also very easy to verify the conditions of Theorem 8 in practice. One just needs to compute the unique $\alpha$ such that $k \mathbb{E}\left(y_{0}-y_{0}^{\alpha}\right)=S_{1}$ and then check the existence of $\tau^{*} \geq 0$ and $z^{*}$ in $\Delta_{\rho}$.
Remark 5. We pointed out in Remark 3 that quota-share-like contracts will not be optimal in practice. However, things are quite different if we deal with stop loss contracts. The conditions of Theorems 7 and 8 may hold in practice, as will be shown in the next section. Unfortunately, it seems that there is no simple economic interpretation of these conditions. From a mathematical point of view, their fulfillment may be related to the existence of bang-bang solutions $z^{*}$ for (14), if stop loss reinsurance contracts are involved. In the next section, examples of bang-bang solutions will be found if $\rho$ is the absolute deviation or the conditional value at risk.

\section{Particular risk functions}

Until now, all the previous results of the paper hold regardless of the risk function we are using. In this section we will analyze some important examples of risk function. In particular, we will focus on the standard deviation since, as indicated in the introduction, it is frequently used in the literature, the absolute deviation, since it has better properties with respect to the second order stochastic dominance if asymmetry and/or heavy tails are involved (Ogryczak and Ruszczynski, 1999), and the conditional value at risk, since it is becoming a very well-known coherent and expectation bounded risk measure that also respects the stochastic dominance (Ogryczak and Ruszczynski, 2002).

In general, the $p$-deviation

$\sigma_{p}: L^{p} \longrightarrow \mathbb{R}$

is defined by

$\sigma_{p}(y)=\left(\mathbb{E}\left(|y-\mathbb{E}(y)|^{p}\right)\right)^{\frac{1}{p}}=\|y-\mathbb{E}(y)\|_{p}$.

Since $L^{q}$ is the dual space of $L^{p}$ it is known that

$$
\begin{aligned}
\sigma_{p}(y) & =\operatorname{Max}\left\{\mathbb{E}((y-\mathbb{E}(y)) z) ; z \in L^{q},\|z\|_{q} \leq 1\right\} \\
& =\operatorname{Max}\left\{\mathbb{E}(y z)-\mathbb{E}(y) \mathbb{E}(z) ; z \in L^{q},\|z\|_{q} \leq 1\right\} \\
& =\operatorname{Max}\left\{\mathbb{E}(y(z-\mathbb{E}(z))) ; z \in L^{q},\|z\|_{q} \leq 1\right\} .
\end{aligned}
$$

Hence

$\Delta_{\rho}=\left\{z-\mathbb{E}(z) ; z \in L^{q},\|z\|_{q} \leq 1\right\}$.

Moreover, in the particular case $p=q=2$, by using the properties of the orthogonal projection of Hilbert spaces it is easy to prove that

$\Delta_{2}=\left\{z ; z \in L^{2},\|z\|_{2} \leq 1, \mathbb{E}(z)=0\right\}$.

(Rockafellar et al., 2006). ${ }^{13}$

Theorem 9. Suppose that $p=2$ and $\rho=\sigma_{2}$.

(a) If there is a zero-variance (constant) (5)-feasible random variable $y^{*}$ then $y^{*}$ solves (5). If so, the optimal value of (5) vanishes.

(b) If there are no zero-variance (5)-feasible random variables and $\alpha>0$ is such that the stop loss reinsurance $y_{0}^{\alpha}$ satisfies $k \mathbb{E}\left(y_{0}-y_{0}^{\alpha}\right)=S_{1}$ then $y_{0}^{\alpha}$ solves (5). ${ }^{14}$

\footnotetext{
13 Obviously

$\Delta_{2} \sqsupset\left\{z ; z \in L^{2},\|z\|_{2} \leq 1, \mathbb{E}(z)=0\right\}$
}

because $z=z-\mathbb{E}(z)$ whenever $\mathbb{E}(z)=0$, and the opposite inclusion holds because for every $z$ in the unit ball of $L^{2}$ we have that $\mathbb{E}(z)$ and $z-\mathbb{E}(z)$ are orthogonal, and therefore the Pithagorean Theorem leads to

$1 \geq\|z\|_{2}^{2}=\|\mathbb{E}(z)\|_{2}^{2}+\|z-\mathbb{E}(z)\|_{2}^{2} \geq\|z-\mathbb{E}(z)\|_{2}^{2}$.

14 This result is closely related to that of Borch (1960). 
Proof. (a) is obvious so let us prove (b). We will prove that the requirements of (15) are fulfilled. Take

$\tau^{*}=\frac{\alpha-\mathbb{E}\left(y_{0}^{\alpha}\right)}{k \sigma_{2}\left(y_{0}^{\alpha}\right)}$

and

$z^{*}=\frac{y_{0}^{\alpha}-\mathbb{E}\left(y_{0}^{\alpha}\right)}{\sigma_{2}\left(y_{0}^{\alpha}\right)}$.

First of all notice that $\sigma_{2}\left(y_{0}^{\alpha}\right)>0$ because $y_{0}^{\alpha}$ is (5)-feasible and there are no zero-variance (5)-feasible random variables. Therefore the definitions above are correct.

Secondly, $\tau^{*} \geq 0$, since $\alpha<\mathbb{E}\left(y_{0}^{\alpha}\right)$ cannot hold. Thirdly, $z^{*} \in \Delta_{2}$. Indeed, according to (28) we must show that $\mathbb{E}\left(z^{*}\right)=0$ and $\left\|z^{*}\right\|_{2}^{2} \leq 1$. The first equality is obvious, whereas the inequality is also satisfied because

$$
\begin{aligned}
\left\|z^{*}\right\|_{2}^{2} & =\left\|\frac{y_{0}^{\alpha}-\mathbb{E}\left(y_{0}^{\alpha}\right)}{\sigma_{2}\left(y_{0}^{\alpha}\right)}\right\|_{2}^{2} \\
& =\frac{\left\|y_{0}^{\alpha}-\mathbb{E}\left(y_{0}^{\alpha}\right)\right\|_{2}^{2}}{\sigma_{2}\left(y_{0}^{\alpha}\right)^{2}}=\frac{\sigma_{2}\left(y_{0}^{\alpha}\right)^{2}}{\sigma_{2}\left(y_{0}^{\alpha}\right)^{2}}=1 .
\end{aligned}
$$

Next let us prove that $\mathbb{E}\left(y_{0}^{\alpha} z^{*}\right) \geq \mathbb{E}\left(y_{0}^{\alpha} z\right)$ for every $z \in \Delta_{2}$. We will show that $z^{*}$ solves the variational problem

$$
\begin{aligned}
& \operatorname{Max} \int_{\Omega} y_{0}^{\alpha} z \mathrm{~d} \mu \\
& \int_{\Omega} z \mathrm{~d} \mu=0 \\
& \int_{\Omega} z^{2} \mathrm{~d} \mu \leq 1 .
\end{aligned}
$$

Since this problem is obviously convex, it is sufficient to show that $z^{*}$ satisfies the Karush-Kuhn-Tucker conditions (Luenberger, 1969), i.e., we must state the existence of $L_{1}, L_{2} \in \mathbb{R}, L_{2} \geq 0$, such that $L_{2}\left(1-\left\|z^{*}\right\|_{2}^{2}\right)=0$ and

$y_{0}^{\alpha}=L_{1}+2 L_{2} z^{*}=L_{1}+2 L_{2} \frac{y_{0}^{\alpha}-\mathbb{E}\left(y_{0}^{\alpha}\right)}{\sigma_{2}\left(y_{0}^{\alpha}\right)}$.

The first condition is obvious because (29) shows that $\left\|z^{*}\right\|_{2}^{2}=$ 1 , and the second one clearly holds for $L_{1}=\mathbb{E}\left(y_{0}^{\alpha}\right)$ and $L_{2}=$ $\frac{1}{2} \sigma_{2}\left(y_{0}^{\alpha}\right)$.

It only remains to verify the second condition of (15) or, equivalently, (17). Take the partition $\Omega_{1}=\emptyset, \Omega_{2}=$ $\left\{\omega \in \Omega ; y_{0}(\omega) \geq \alpha\right\}$ and $\Omega_{3}=\left\{\omega \in \Omega ; y_{0}(\omega)<\alpha\right\}$, and the fulfillment of $(17)$ is trivial.

Remark 6. According to the theorem above, the optimal retention will always be a stop loss contract saturating the reinsurance price constraint, unless there exist zero-variance feasible retentions. ${ }^{15}$ Despite the fact that this mathematical finding must be pointed out, it is obvious that the existence of zero-variance feasible retentions will never hold in practice.

Remark 7. In the proof of the theorem above, we have provided the values of $\tau^{*}$ and $z^{*}$ without any previous computation, and then we have checked that $z^{*}$ solves the variational problem (14). However, in practice, the process will be different, that is, we will

\footnotetext{
15 Remark 9 will introduce a minor nuance for some particular cases.
}

have to solve (14) in order to establish the relationship between the primal solution $y^{*}$ and the dual one $z^{*}$. For this reason, we will follow this second method in order to study the absolute deviation and the conditional value at risk, despite the fact that the exposition will be a little bit more tedious.

Remark 8. Let us now consider Problem (5) with $\rho=\sigma_{1}$. Then (27) obviously implies that

$\Delta_{1}=\left\{z-\mathbb{E}(z) ; z \in L^{\infty},-1 \leq z \leq 1\right\}$.

Then the variational principle of Theorem 4 and Remark 2 lead to the linear optimization problem

$$
\left\{\begin{array}{l}
\operatorname{Max} \mathbb{E}(y(z-\mathbb{E}(z)))=\int_{\Omega} y z \mathrm{~d} \mu-\mathbb{E}(y) \int_{\Omega} z \mathrm{~d} \mu \\
z \leq 1 \\
z \geq-1 \\
z \in L^{\infty} .
\end{array}\right.
$$

It is easy to verify that the problem above satisfies the Slater qualification (Luenberger, 1969), so the Karush-Kuhn-Tucker conditions become necessary and sufficient. Furthermore, the dual space of $L^{\infty}$ contains $L^{1}$ and is composed of those finitely-additive measures on the $\sigma$-algebra $\mathcal{F}$ of $\Omega$ being $\mu$-continuous and having finite variation (Horvàth, 1966). Thus, the Karush-Kuhn-Tucker conditions lead to the existence of two such measures $m_{1} \geq 0$ and $m_{2} \geq 0$ such that

$\left\{\begin{array}{l}y=\mathbb{E}(y)+m_{1}-m_{2} \\ \int_{\Omega}(1-z) \mathrm{d} m_{1}=0 \\ \int_{\Omega}(1+z) \mathrm{d} m_{2}=0 .\end{array}\right.$

The second and third conditions lead to $z=1$ whenever $m_{1} \neq 0$ and $z=-1$ whenever $m_{2} \neq 0$. Thus, there is a measurable partition $\Omega=A \cup B \cup C$ such that

$\left\{\begin{array}{l}z=-1, \quad m_{1}=0, \quad \omega \in A \\ -1 \leq z \leq 1, \quad m_{1}=m_{2}=0, \quad \omega \in B \\ z=1, \quad m_{2}=0, \quad \omega \in C .\end{array}\right.$

Consequently, the first equality in (30) gives

$\begin{cases}m_{2}=\mathbb{E}(y)-y, & \omega \in A \\ m_{1}=y-\mathbb{E}(y) & \omega \in C\end{cases}$

and therefore $m_{i} \in L^{1}, i=1,2$, because they vanish out of the indicated sets. Summarizing

$\left\{\begin{array}{l}z=-1, \quad m_{1}=0, \quad y=\mathbb{E}(y)-m_{2} \leq \mathbb{E}(y), \quad \omega \in A \\ -1<z<1, \quad m_{1}=m_{2}=0, \quad y=\mathbb{E}(y), \quad \omega \in B \\ z=1, \quad m_{2}=0, \quad y=\mathbb{E}(y)+m_{1} \geq \mathbb{E}(y), \quad \omega \in C .\end{array}\right.$

If we assume that $\mu(y>0)=1$ the remaining conditions in Theorem 4 impose the existence of $\tau \geq 0$ such that (see also Lemma 5 and recall that $\tilde{E}=0$ for deviations)

$\left\{\begin{array}{l}z \leq \mathbb{E}(z)+k \tau \\ z=\mathbb{E}(z)+k \tau, \quad \text { if } y<y_{0} .\end{array}\right.$

Therefore, an upper bound of $z$ will be $\mathbb{E}(z)+k \tau$. Since $z \leq 1$ there are three cases to consider:

Case 1. $\mathbb{E}(z)+k \tau>1$. Then (31) and (32) imply that $y=y_{0}$ Thus, the fourth condition of (13) is not saturated, which implies that $\tau=0$. Then $z \leq \mathbb{E}(z)$ implies $z=\mathbb{E}(z)$, i.e., $z$ is constant. Hence one and only one set in the partition $\Omega=A \cup B \cup C$ is non void. If $\Omega=A$ then $y \leq \mathbb{E}(y)$ leads to $y=\mathbb{E}(y)$, i.e., $y_{0}=\mathbb{E}\left(y_{0}\right)$. Similarly, the conclusion $y_{0}=\mathbb{E}\left(y_{0}\right)$ is also achieved if $\Omega=B$ or $\Omega=C$, so $y=y_{0}$ and $y_{0}$ is constant. 
Case 2. $\mathbb{E}(z)+k \tau<1$. In such a case $C$ is void (or a null set) and (31) implies that $y \leq E(y)$. Thus $y=E(y)$ has to be constant.

Case 3. $\mathbb{E}(z)+k \tau=1$. Bearing in mind (31) there must be a measurable set $C$ such that

$$
\begin{cases}y=y_{0}, & \omega \notin C \\ \mathbb{E}(y) \leq y<y_{0}, & \omega \in C \\ k \mathbb{E}\left(y_{0}-y\right)=S_{1} & \end{cases}
$$

and these are the necessary and sufficient conditions so as to guarantee that $y$ is optimal, since the requirements of (15) and (17) are fulfilled by taking $z^{*}=z-\mathbb{E}(z)$ with $z=\chi_{C}-\chi_{\Omega \backslash C}$ and $\tau^{*}=\frac{1-\mathbb{E}(z)}{k}$, which is non negative because $-1 \leq z \leq 1$ and satisfies

$z^{*}=z-\mathbb{E}(z) \leq 1-\mathbb{E}(z)=k \tau^{*}$

with equality on $C$ because $z=1 .{ }^{16}$

In order to summarize, we will provide a formal statement reflecting the findings above.

Theorem 10. Suppose that $p=1$ and $\rho=\sigma_{1}$.

(a) If there exists a zero-variance (constant) (5)-feasible random variable $y^{*}$ then $y^{*}$ solves (5). If so, the optimal value of (5) vanishes.

(b) If there are no zero-variance (5)-feasible random variables and $y^{*}$ is (5)-feasible and such that $\mu\left(y^{*}>0\right)=1$ then $y^{*}$ solves (5) if and only if there exists a measurable set $C$ such that (33) holds.

(c) If there are no zero-variance (5)-feasible random variables and $\alpha>0$ is such that $y_{0}^{\alpha}$ satisfies

$k \mathbb{E}\left(y_{0}-y_{0}^{\alpha}\right)=S_{1}$

then $y_{0}^{\alpha}$ solves (5).

Proof. (a) is obvious and (b) may be proved by checking that the elements $z^{*}$ and $\tau^{*}$ in the remark above make Conditions (15) hold To prove (c) one must show that the conditions of (b) are respected by $y_{0}^{\alpha}$. Take

$C=\left\{\omega \in \Omega ; y_{0}(\omega)>\alpha\right\}$,

and (b) becomes obvious.

Remark 9. Comments of Remark 6 also apply here, i.e., the (5)feasible stop loss reinsurance saturating the reinsurance price constraint will be the optimal retention in practical situations. According to Theorem 10(b), "more sophisticated" contracts might be optimal too. They are "almost" stop loss reinsurance, or "close" to a stop loss reinsurance, but they do not have to be an exact stop loss reinsurance. Nevertheless, if there were no uniqueness in the solution of (5) it seems to be clear that agents would choose the stop loss solution.

Remark 10. Suppose now that $\rho=C V a R_{\mu_{0}}$ with $\mu_{0} \in(0,1)$ being the level of confidence. ${ }^{17}$ In such a case Rockafellar et al. (2006) has stated that

$\Delta_{\rho}=\left\{z \in L^{\infty} ; 0 \leq z \leq \frac{1}{\mu_{0}}, \mathbb{E}(z)=1\right\}$.

\footnotetext{
16 As usual, $\chi_{C}$ and $\chi_{\Omega \backslash C}$ are the characteristic functions of $C$ and its complementary $\Omega \backslash C$

17 In order to simplify the exposition we will assume that $k<1 / \mu_{0}$ and that the distribution of $y_{0}$ is continuous. The rest of the cases may also be analyzed but the exposition is much more tedious. Furthermore, both restrictions are quite natural. In particular, regarding the first one, $k$ will never in practice be higher than 2 or 3 , and $\mu_{0}$ will never be more than $5 \%$, i.e., $1 / \mu_{0}$ will be 20 at least.
}

Consequently, if $\mu(y>0)=1$ and we would like to check whether $y$ solves (5) then the variational principle (14) suggests solving the linear optimization problem

$\left\{\begin{array}{l}\operatorname{Max} \mathbb{E}(y z)=\int_{\Omega} y z \mathrm{~d} \mu \\ z \leq 1 / \mu_{0} \\ z \geq 0 \\ \int_{\Omega} z \mathrm{~d} \mu=1 \\ z \in L^{\infty} .\end{array}\right.$

Once again it is easy to verify the fulfillment of the Slater qualification, and the Karush-Kuhn-Tucker conditions become

$\left\{\begin{array}{l}y=L+m_{1}-m_{2} \\ \int_{\Omega}\left(1 / \mu_{0}-z\right) \mathrm{d} m_{1}=0 \\ \int_{\Omega} z \mathrm{~d} m_{2}=0 \\ L \in \mathbb{R}, m_{1} \geq 0, m_{1} \geq 0 .\end{array}\right.$

As in the previous case, we can find a partition $\Omega=A \cup B \cup C$ such that

$\left\{\begin{array}{l}z=0, \quad m_{1}=0, \quad y=L-m_{2} \leq L, \quad \omega \in A \\ 0<z<1 / \mu_{0}, \quad m_{1}=m_{2}=0, \quad y=L, \quad \omega \in B \\ z=1 / \mu_{0}, \quad m_{2}=0, \quad y=L+m_{1} \geq L, \quad \omega \in C\end{array}\right.$

and $m_{1}$ and $m_{2}$ become random variables of $L^{1}$. In addition, since $\tilde{E}=1$ in this case, (17) implies the existence of $\tau \geq 0$ such that $z \leq k(1+\tau), z=k(1+\tau)$ whenever $y<y_{0}$, and $\tau\left(k \mathbb{E}\left(y_{0}-y\right)-S_{1}\right)=0$. Let us consider three possible scenarios: Case $1, k(1+\tau)=1 / \mu_{0}$. Then $\tau=\frac{1}{k \mu_{0}}-1>0$ and therefore $k \mathbb{E}\left(y_{0}-y\right)-S_{1}=0$. Moreover $\mu(B)=0$ since otherwise $z<$ $k(1+\tau)$ on $B$ leads to $y_{0}=y=L$ on $B$ and $y_{0}$ cannot be constant with positive probability because its distribution is continuous. Thus, let us remove $B$ from (36). $\mathbb{E}(z)=1$ implies that $\mu(C)=$ $\mu_{0}$, so the necessary and sufficient conditions guaranteeing that $y$ solves (5) will be: $k \mathbb{E}\left(y_{0}-y\right)-S_{1}=0$ and there exists a measurable set $C$ such that $\mu(C)=\mu_{0}, y=y_{0}$ out of $C$, and $y_{0}\left(\omega_{C}\right) \geq y_{0}(\omega)$ whenever $\omega_{C} \in C$ and $\omega \notin C$. In particular, if $\alpha$ is such that $k \mathbb{E}\left(y_{0}-y_{0}^{\alpha}\right)-S_{1}=0$ then $y_{0}^{\alpha}$ satisfies the conditions of this case if and only if $\mu\left(y_{0}>\alpha\right) \leq \mu_{0}$, since in such a case we can extend $\left\{y_{0}>\alpha\right\}$ to a set $C=\left\{y_{0}>\alpha^{\prime}\right\}\left(\alpha^{\prime} \leq \alpha\right)$ such that $\mu\left(y_{0}>\alpha\right)=\mu_{0}$ (recall that $y_{0}$ has continuous distribution).

Case $2, k(1+\tau)<1 / \mu_{0}$. In this second scenario $C$ has null probability, so let us remove it in (36). Then (36) clearly points out that $y=y_{0}^{L}$, since $z=0<k(1+\tau)$ on $A$ leads to $y=y_{0}$ on $A$. Notice that three more requirements must be satisfied. Firstly, $k \mathbb{E}\left(y_{0}-y_{0}^{L}\right)-S_{1} \leq 0$, secondly, $\mathbb{E}(z)=1$ implies $\mu(B) k(1+\tau) \geq$ 1 , and thus

$1 / \mu(B) \leq k(1+\tau)<1 / \mu_{0}$

which also leads to $\mu_{0}<\mu(B)$. Furthermore, $z$ must be constant (and equal $k(1+\tau)$ ) on $B$, because otherwise $y_{0}=y_{0}^{L}=L$ on a subset of $B$ with positive probability, and the distribution of $y_{0}$ being continuous we have $\mu\left(y_{0}=L\right)=0$. Then, $\mu(B) k(1+\tau)=1$ implies $0 \leq \tau=\frac{1}{k \mu(B)}-1$, and $1 / \mu(B) \geq k$ must hold. Thirdly, $\tau\left(k \mathbb{E}\left(y_{0}-y_{0}^{L}\right)-S_{1}\right)=0$ provokes that $k \mathbb{E}\left(y_{0}-y_{0}^{L}\right)=S_{1}$ or $1 / \mu(B)=k$.

Case $3, k(1+\tau)>1 / \mu_{0}$. Then (36) shows that $z=k(1+\tau)$ never holds, and then $y=y_{0}$. On the other hand $\tau>\frac{1}{k \mu_{0}}-1>0$ implies $0=k \mathbb{E}\left(y_{0}-y\right)=S_{1}$, contradicting the assumptions. 
Theorem 11. Suppose that the distribution of $y_{0}$ is continuous, $p=$ 1, $\rho=\mathrm{CVaR}_{\mu_{0}}$ with $0<\mu_{0}<1,1 / \mu_{0}>k$ and $y^{*}$ is a feasible solution such that $\mu\left(y^{*}>0\right)=1$. Then, $y^{*}$ solves (5) if and only if at least one of the following assertions hold:

(a) $k \mathbb{E}\left(y_{0}-y^{*}\right)=S_{1}$ and there exists a measurable set $C$ such that $\mu(C)=\mu_{0}, y^{*}=y_{0}$ out of $C$, and

$y_{0}\left(\omega_{\mathrm{C}}\right) \geq y^{*}\left(\omega_{\mathrm{C}}\right) \geq y^{*}(\omega)=y_{0}(\omega)$

whenever $\omega_{C} \in C$ and $\omega \notin C$. In such a case the optimal value of (5) is

$\mathbb{E}\left(y^{*} z^{*}\right)-\left(S_{0}-k \mathbb{E}\left(y_{0}-y^{*}\right)\right)$,

where $z^{*}=\frac{1}{\mu_{0}} \chi_{c}$.

(b) $y^{*}=y_{0}^{\alpha}$ and $\alpha>0$ is such that $k \mathbb{E}\left(y_{0}-y_{0}^{\alpha}\right)=S_{1}$ and $\mu\left(y_{0}>\alpha\right) \leq \mu_{0}$. In such a case the optimal value of (5) is (37), where $z^{*}=\frac{1}{\mu_{0}} \chi_{c}, C$ being a set of the form $C=\left\{\omega ; y_{0}>\alpha^{\prime}\right\}$ for some $\alpha^{\prime} \leq \alpha$ and such that $\mu(C)=\mu_{0}$

(c) $y^{*}=y_{0}^{\alpha}, \alpha>0, k \mathbb{E}\left(y_{0}-y_{0}^{\alpha}\right)=S_{1}, \frac{1}{\mu\left(y_{0}>\alpha\right)} \geq k$ and $\mu_{0}<\mu\left(y_{0}>\alpha\right)$. If so the optimal value of (5) is (37), with $z^{*}=$ $\frac{1}{\mu\left(y_{0}>\alpha\right)} \chi_{\mu\left(y_{0}>\alpha\right)}$.

(d) $y^{*}=y_{0}^{\alpha}, \alpha>0, k \mathbb{E}\left(y_{0}-y_{0}^{\alpha}\right) \leq S_{1}, \frac{1}{\mu\left(y_{0}>\alpha\right)}=k$ and $\mu_{0}<\mu\left(y_{0}>\alpha\right)$. If so, the optimal value of (5) is (37), where $z^{*}=\frac{1}{\mu\left(y_{0}>\alpha\right)} \chi_{\mu\left(y_{0}>\alpha\right)}$.

Proof. It easily follows from the previous remark and the equalities (16) and $\tilde{E}=1$.

Remark 11. The theorem above again points out that the stop loss reinsurance with $k \mathbb{E}\left(y_{0}-y_{0}^{\alpha}\right)=S_{1}$ will frequently be optimal. According to Statements (b) and (c) this occurs if $\mu\left(y_{0}>\alpha\right) \leq \mu_{0}$ or

$\mu_{0}<\mu\left(y_{0}>\alpha\right) \leq \frac{1}{k}$,

which probably hold. If they did not hold, i.e., if

$\mu\left(y_{0}>\alpha\right)>\frac{1}{k}$

one can look for a different stop loss optimal solution. Indeed, (38) is impossible in the limit case $k=1$, though it becomes more probable as $k$ increases. If $k$ is high enough and (38) holds then $y_{0}^{\alpha}$ becomes "too expensive", and the risk (see (5))

$\mathrm{CVaR}_{\mu_{0}}\left(S_{0}-y_{0}^{\alpha}-k \mathbb{E}\left(y_{0}-y_{0}^{\alpha}\right)\right)$

becomes "too high", since it obviously increases when $k$ increases. Then take $\alpha^{*}>\alpha$ so as to reach

$\mu\left(y_{0}>\alpha^{*}\right)=\frac{1}{k}$.

The reinsurance price constraint of (5) will not be saturated by the cheaper contract $y_{0}^{\alpha^{*}}$. Furthermore, $y_{0}^{\alpha^{*}}$ satisfies the conditions of Theorem 11(d), so it is the optimal retention.

Finally, notice that, according to (a) and (b), if $y_{0}^{\alpha}$ saturates the reinsurance price constraint and

$\mu\left(y_{0}>\alpha\right) \leq \mu_{0}$

then (for reasonable values of $\mu_{0}$ it is not very probable) the uniqueness of the solution $y_{0}^{\alpha}$ is not guaranteed. A more sophisticated contract could be optimal too. However, as in Remark 9, it seems natural that agents would choose $y_{0}^{\alpha}$.

\section{Conclusions}

The optimal reinsurance problem is a classic topic in Actuarial Theory and has been studied under different assumptions and by using different criteria to compute the insurer risk. Furthermore, general risk measures are becoming very important in Finance and Insurance, and many classic problems have been revisited, taking this approach into account. This article has shown that the optimal reinsurance problem may be analyzed by drawing on general risk measures such as deviation measures, expectation bounded measures of risk or coherent measures of risk, among others. A unified approach has been presented, in the sense that the findings are general enough and do not depend on the concrete risk measure to be used. Necessary and sufficient optimality conditions have been provided. These conditions have been used so as to study the most important types of reinsurance in practice, pointing out that quota-share-like reinsurance contracts can barely be optimal. Furthermore, three important concrete risk measures have been analyzed in detail, with special focus on the conditional value at risk, since this coherent and expectation bounded risk measure is being used more and more in Finance and Insurance due to its interesting properties.

\section{Acknowledgments}

The authors are sincerely grateful for the comments and suggestions of the anonymous reviewer, which have led to significant improvements of the paper.

Research partially developed during the sabbatical visit to Concordia University (Montreal, Québec, Canada). Alejandro and Beatriz Balbás would like to thank the Department of Mathematics and Statistics for its great hospitality, in particular José Garrido and Yogendra Chaubey.

Research partially supported by "Welzia Management SGIIC SA", "RD_Sistemas SA", "Comunidad Autónoma de Madrid" (Spain), Grant s-0505/tic/000230, and "MEyC" (Spain), Grant SEJ2006-15401C04.

The usual caveat applies.

\section{References}

Alexander, S., Coleman, T.F., Li, Y., 2006. Minimizing CVaR and VaR for a portfolio of derivatives. Journal of Banking \& Finance 30, 538-605.

Arrow, K.J., 1963. Uncertainty and the welfare of medical care. American Economic Review 53, 941-973.

Artzner, P., Delbaen, F., Eber, J.M., Heath, D., 1999. Coherent measures of risk. Mathematical Finance 9, 203-228.

Balbás, A., Balbás, R., Mayoral, S., 2009. Portfolio choice problems and optimal hedging with general risk functions: A simplex-like algorithm. European Journal of Operational Research 192 (2), 603-620.

Balbás, A., Romera, R., 2007. Hedging bond portfolios by optimization in Banach spaces. Journal of Optimization Theory and Applications 132 (1), 175-191.

Basak, S., Shapiro, A., 2001. Value-at-risk-based risk management: Optimal policies and asset prices. Review of Financial Studies 14, 371-405.

Borch, K., 1960. An attempt to determine the optimum amount of stop loss reinsurance. In: Transactions of the 16th International Congress of Actuaries I, 597-610.

Bühlmann, H., 1970. Mathematical Methods in Risk Theory. Springer Verlag, Berlin.

Burgert, C., Rüschendorf, L., 2006. Consistent risk measures for portfolio vectors. Insurance: Mathematics and Economics 38 (2), 289-297.

Cai, J., Tan, K.T., 2007. Optimal retention for a stop loss reinsurance under the VaR and CTE Risk Measures. ASTIN Bulletin 37 (1), 93-112.

Carr, P., Heman, H., Madan, D., 2001. Pricing and hedging in incomplete markets. Journal of Financial Economics 62, 131-167.

Dhaene, J., Laeven, R., Vanduffel, S., Darkiewicz, G., Goovaerts, M., 2008. Can a coherent risk measure be too subadditive?. Journal of Risk and Insurance 75, 365-386.

Denneberg, D., 1994. Non-Additive Measure and Integral. Kluwer Academic Publishers, Boston.

Deprez, O., Gerber, U., 1985. On convex principles of premium calculation. Insurance: Mathematics and Economics 4, 179-189.

Frittelli, M., Scandolo, G., 2005. Risk measures and capital requirements for processes. Mathematical Finance 16 (4), 589-612.

Gajec, L., Zagrodny, D., 2004. Optimal reinsurance under general risk measures. Insurance: Mathematics and Economics 34, 227-240.

Gerber, H., 1979. An introduction to mathematical risk theory. Huebner Foundation Monograph 8. Homewood, IL. 
Goovaerts, M., Etienne, F., De Vylder, C., Haezendonck, J., 1984. Insurance Premiums. North-Holland Publishing, Amsterdam.

Goovaerts, M., Kaas, R., Dhaene, J., Tang, Q., 2004a. A new classes of consistent risk measures. Insurance: Mathematics and Economics 34, 505-516.

Goovaerts, M. Kaas, R, Laeven, R, Tang, Q, 2004b. A comonotonic image of independence for additive risk measures. Insurance: Mathematics and Economics 35, 581-594.

Goovaerts, M., Laeven, R., 2008. Actuarial risk measures for financial derivative pricing. Insurance: Mathematics and Economics 42, 540-547.

Horvàth, J., 1966. Topological Vector Spaces and Distributions, vol. I. Addison Wesley, Reading, MA.

Huber, P., 1981. Robust Statistics. Wiley, New York.

Jin, H., Xu, Z.Q., Zhou, X.Y., 2008. A convex stochastic optimization problem arising from portfolio selection. Mathematical Finance 18, 171-183.

Kaluszka, M., 2001. Optimal reinsurance under mean-variance premium principles. Insurance: Mathematics and Economics 28, 61-67.

Kaluszka, M., 2005. Optimal reinsurance under convex principles of premium calculation. Insurance: Mathematics and Economics 36, 375-398.

Laeven, R., Goovaerts, M., 2004. An optimization approach to the dynamic allocation of economic capital. Insurance: Mathematics and Economics 35, 299-319.

Luenberger, D.G., 1969. Optimization by Vector Spaces Methods. John Wiley \& Sons, New York.
Mansini, R., Ogryczak, W., Speranza, M.G., 2007. Conditional value at risk and related linear programming models for portfolio optimization. Annals of Operations Research 152, 227-256.

Nakano, Y., 2004. Efficient hedging with coherent risk measure. Journal of Mathematical Analysis and Applications 293, 345-354.

Ogryczak, W., Ruszczynski, A., 1999. From stochastic dominance to mean risk models: Semideviations and risk measures. European Journal of Operational Research 116, 33-50.

Ogryczak, W., Ruszczynski, A., 2002. Dual stochastic dominance and related mean risk models. SIAM Journal on Optimization 13, 60-78.

Rockafellar, R.T., Uryasev, S., Zabarankin, M., 2006. Generalized deviations in risk analysis. Finance \& Stochastics 10, 51-74.

Schied, A., 2007. Optimal investments for risk- and ambiguity-averse preferences: A duality approach. Finance \& Stochastics $11,107-129$.

Schmeidler, D., 1986. Integral representation without additivity. Proceedings of the American Mathematical Society 97, 255-261.

Wang, S.S., 2000. A class of distortion operators for pricing financial and insurance risks. Journal of Risk and Insurance 67, 15-36.

Young, V.R., 1999. Optimal insurance under Wang's premium principle. Insurance: Mathematics and Economics 25, 109-122.

Zalinescu, C., 2002. Convex Analysis in General Vector Spaces. World Scientific Publishing Co. 